\title{
Percepção da qualidade de vida e da finitude de adultos de meia idade e idoso praticantes e não praticantes de atividade física
}

\author{
Perception of quality of life and finitude of middle-aged adults and elderly practitioners and non- \\ practitioners of physical activity
}

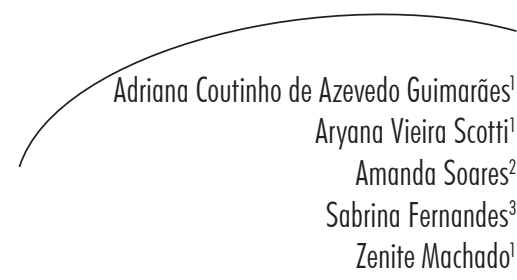

Resumo

Objetivo: Este estudo teve como objetivo descobrir a influência da atividade física na percepção de qualidade de vida $(\mathrm{QV})$ e finitude de indivíduos praticantes e não praticantes de atividade física e de dança em salões de dança da grande FlorianópolisSC. Métodos: Participaram por conveniência 195 indivíduos com média de idade de

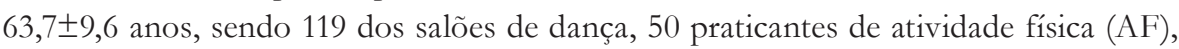
e 26 que não realizam AF. Optou-se por um questionário autoaplicável composto de instrumentos previamente validados (Whoqol abreviado e Finitude de Sheppard). A análise foi descritiva e inferencial por meio do teste do Qui-quadrado e ANOVA. Resultados: A qualidade de vida pode ser considerada positiva com escores que variaram de 70,3 a 58,9\% entre seus domínios, tendo uma percepção positiva da finitude, no qual o grupo que realiza dança $(79,8 \%)$ destacou-se em relação aos demais $(p=0,035)$. Conclusão: De acordo com os resultados, entende-se que a prática da dança como AF pode contribuir na percepção positiva da QV e na finitude.

\section{Abstract}

Objective: This study aimed to investigate the influence of physical activity on perceived quality of life (QOL) and finitude of individuals practicing and non-practicing physical activity and dance in the dance halls of Florianopolis city, state of Santa Catarina, Brazil. Methods: Participants were 195 individuals for convenience with a mean age of $63.7 \pm 9.6$ years, 119 of the dance halls, 50 physically active (AF), and 26 who did not perform AF. We opted for a self-administered questionnaire composed of previously validated instruments (abbreviated Whoqol and Finitude by Sheppard). The analysis was descriptive and inferential through Chi square test and ANOVA. Results: The quality of life can be considered positive with scores ranging from 70.3 to $58.9 \%$ between their

Palavras chave: Dança. Terapia pela dança. Qualidade de Vida. Atividade Física. Finitude. Florianópolis - SC.
Key words: Dance. Therapy for dance. Quality of Life. Physical Activity. Finiteness. Florianópolis - SC.

\footnotetext{
Departamento de Educação Física, Centro de Ciências da Saúde e do Esporte. Universidade do Estado de Santa Catarina. Florianópolis, SC, Brasil.

2 Departamento de Fisioterapia, Instituição de Ensino Superior e Departamento de Educação Física. Faculdade Porto das Águas. Porto Belo, SC, Brasil.

3 Programa de Pós-graduação em Atividade Física e Saúde. Universidade Federal de Santa Catarina. Florianópolis, SC, Brasil.
} 
domains, having a positive perception of finitude, in which the group performs dance $(79.8 \%)$ stood out in relation to the others $(\mathrm{p}=0.035)$. Conclusion: According to the results, it is understood that the practice of dance as AF can contribute to the positive perception of QOL and finitude.

\section{INTRODUÇÃOO}

No Brasil atualmente, a população com mais de 60 anos representa cerca de 7\%. No último censo, Sul e Sudeste se mantiveram como as duas regiões mais envelhecidas do país, com aproximadamente $8 \%$ de idosos, mas as projeções mostram que esta proporção nacional aumentará para $23 \%$ em 2050..$^{1}$ A principal razão dessa alteração demográfica em relação às demais faixas etárias é a redução da mortalidade e da fecundidade. ${ }^{2}$

O processo de envelhecimento é caracterizado por mudanças, as quais podem ser traduzidas em diminuição da plasticidade comportamental, em aumento da vulnerabilidade e da probabilidade de morte. ${ }^{3}$ Este processo exige uma adaptação às novas condições de vida, um reposicionamento social e aprendizagem para usar o tempo livre, além de modificação na maneira de se situar e agir. ${ }^{4}$

A consciência da temporalidade é um problema recorrente no processo de envelhecimento, sendo que alguns temas, como a morte e finitude, se tornam mais frequentes nesta etapa da vida. O processo que leva até a morte é um fato irrefutável perante os sentidos imediatos, em que vida e morte devem ser consideradas como duas faces da existência humana. ${ }^{5,6}$

À medida que o ser humano toma consciência de sua finitude, passa a compreender a vida em sua complexidade e tende a rever seus valores. A contextualização do processo de envelhecimento e morte em suas dimensões sociais, culturais, psicológicas e espirituais possibilita ao idoso um ambiente para expressar seus sentimentos e o seu modo de ser e ver o mundo. ${ }^{7,8}$

Com a consciência de finitude, novas atitudes e mudanças de hábitos auxiliam no processo de envelhecimento. Nomeadamente na atividade física, tem-se um importante recurso para minimizar as perdas provocadas por este processo, podendo aumentar ou manter a aptidão física da população idosa, potencializando o bem-estar funcional e melhorando sua qualidade de vida, ${ }^{9}$ que está definitivamente ligada ao grau de satisfação do indivíduo em todos os sentidos da vida pessoal. ${ }^{10}$

Como existem várias formas de ser ativo fisicamente, chama-se a atenção para a dança, que é uma modalidade de atividade física que pode estar totalmente voltada aos idosos e também tem como objetivo aumentar o condicionamento físico, o ritmo, a flexibilidade, a força e a leveza, além de ser extremamente prazerosa ${ }^{11}$ e bem aceita por este público. ${ }^{12}$ É uma atividade lúdica, de manifestação artística, uma comunicação praticada com o corpo; quando exercitada em grupo, ajuda a expressar as emoções, estimulando a memorização e a coordenação. ${ }^{13}$

No Brasil, especialmente na Região Sul, a população a partir da meia idade possui uma empatia pelos bailes, o que faz aumentar vertiginosamente a procura por salões de dança. ${ }^{10}$ Estes constituem um espaço de possibilidades além do ambiente doméstico, num lazer desvinculado da família e do lar. ${ }^{14}$ Estudiosos $^{15}$ afirmam ainda que os bailes englobam uma variedade de ritmos e músicas, que atende às necessidades e exigências de todos os frequentadores, onde a combinação de movimentos explora a criatividade, a capacidade de imaginação e cognição.

Contudo, ainda são escassos os estudos que abordam a temática finitude e a prática de atividade física. Os estudos abordam mais amplamente temas relacionados a ética, ${ }^{16}$ saúde pública, ${ }^{6}$ enfermagem ${ }^{17}$ e medicina,${ }^{5}$ e não fazem uma reflexão direta com a atividade física, 
especialmente com a dança. A relevância científica e social deste estudo consiste em apresentar um novo olhar acerca da finitude relacionada ao processo de envelhecimento. Assim, este estudo investigou a percepção de qualidade de vida e finitude de indivíduos praticantes e não praticantes de atividade física e os que praticam a dança em salões da grande Florianópolis-SC.

\section{METODOLOGIA}

Este é um estudo de corte transversal que foi aprovado pelo Comitê de Ética em Pesquisa em Seres Humanos da Universidade do Estado de Santa Catarina, em 26 de novembro de 2009 (registro no 245/2009). A amostra nãoprobabilística por conveniência foi constituída por 195 indivíduos praticantes e não praticantes de dança em salões, com média de idade de

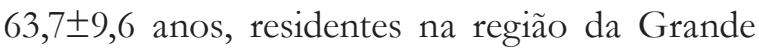
Florianópolis-SC, sendo 119 dos salões de dança, 50 praticantes de atividade física (AF), e 26 que não realizam AF. Os sujeitos não praticantes de dança foram contatados em grupos de convivência da região mencionada. Para atender ao objetivo proposto neste estudo, a amostra foi separada em três grupos: a) grupo formado pelos praticantes de dança nos salões (119 pessoas); b) grupo composto pelos indivíduos que praticavam algum tipo de atividade física (como caminhada, natação, hidroginástica, entre outras - 50 pessoas), menos dança; e c) grupo constituído pelos que não praticam atividade física (26 pessoas). Todos os participantes assinaram o termo de consentimento livre e esclarecido, concordando com a participação no estudo.

Para a coleta de dados foi utilizado um questionário autoaplicável, composto de instrumentos previamente validados:

- informações gerais: sexo, faixa etária, estado conjugal, ocupação profissional e estrato econômico, ${ }^{18}$ totalizando estratos A, B, C, e D;

- características da dança: frequência e permanência nos salões de dança;
- características da atividade física além da dança: realização da atividade física, frequência semanal, tipo e duração;

- qualidade de vida Whoqol abreviado: composta por 26 questões. Esta versão abreviada é composta por quatro domínios: físico, psicológico, social e ambiental, sendo o resultado por escores. Estes variam de $0 \mathrm{a}$ 100 - quanto mais próximo de 100, melhor a qualidade de vida: ${ }^{19}$

- o Questionário sobre Finitude ${ }^{20}$ é um instrumento composto por 20 questões, divididas em quatro subgrupos, os quais permitem avaliar a opinião do respondente quanto a: a) possibilidade de ser feliz na velhice; b) se a velhice prenuncia dependência, morte e solidão; c) se é melhor morrer cedo do que sentir angústia e a solidão da velhice; d) se a velhice pode propiciar sentimentos de integridade. $\mathrm{O}$ questionário é organizado em uma escala do tipo Likert de cinco pontos ("discordo muitíssimo" a "concordo muitíssimo") que permite dupla exploração dos resultados. Num primeiro momento, através de escores, é possível determinar se os participantes possuem uma percepção positiva ou negativa em relação à finitude (prevalência). Posteriormente, reorganizando a pontuação de 10 das 20 questões, pode-se calcular a média em cada um dos subgrupos sendo que quanto mais próxima de 1 (pontuação mínima) mais positiva a percepção do sujeito, e quanto mais próxima de 5 (pontuação máxima), mais negativa.

O instrumento era autoaplicável, e os questionários foram entregues e supervisionados pelos pesquisadores na entrada dos três maiores salões de dança da Grande Florianópolis-SC, e nos grupos de convivência, localizados nos mesmos bairros dos salões de dança, para inquirir indivíduos que realizavam apenas uma (que não a dança) ou nenhuma atividade física.

Os dados foram analisados pelo SPSS - 17.0, através da estatística descritiva (média, desvio padrão, amplitude e percentual) e inferencial 
pelo teste do Qui-quadrado (comparação da percepção positiva e negativa da finitude com os grupos (sem AF, um tipo de AF e dança) e ANOVA (domínios da finitude, nomeadamente felicidade e velhice, dependência e velhice, morte e velhice e integridade e velhice com os três grupos). O nível de significância estatística foi estabelecido para $\mathrm{p}<0.05$.

\section{RESULTADOS}

A tabela 1 evidencia que a maioria dos participantes da amostra é do sexo feminino, praticantes de dança, idosos, com ensino fundamental completo, morando sem companheiros, ainda exercendo alguma atividade profissional e pertencente ao estrato econômico B.

Tabela 1 - Caracterização da amostra quanto à prática da atividade física. Florianópolis-SC, 2010.

\begin{tabular}{|c|c|c|c|}
\hline & $\%$ & $\%$ & $\%$ \\
\hline Variáveis & $\begin{array}{l}\text { Não realiza Atividade Física } \\
\qquad \mathrm{n}=26\end{array}$ & $\begin{array}{l}\text { Atividade Física } \\
\quad \mathrm{n}=50\end{array}$ & $\begin{array}{l}\text { Dança } \\
\mathrm{n}=119\end{array}$ \\
\hline Sexo & $(\%)$ & $(\%)$ & $(\%)$ \\
\hline Masculino & 15,4 & 18,0 & 42,9 \\
\hline Feminino & 84,6 & 82,0 & 57,1 \\
\hline \multicolumn{4}{|l|}{ Idade } \\
\hline Meia idade & 23,1 & 18,0 & 38,7 \\
\hline Idoso & 76,9 & 82,0 & 61,3 \\
\hline \multicolumn{4}{|l|}{ Escolaridade } \\
\hline Ensino fundamental & 57,7 & 46,0 & 39,5 \\
\hline Ensino médio & 23,1 & 40,0 & 45,4 \\
\hline Ensino superior & 19,2 & 8,0 & 11,8 \\
\hline Pós-graduação & 0 & 6,0 & 3,3 \\
\hline \multicolumn{4}{|l|}{ Estado Conjugal } \\
\hline Mora c/ companheiro & 38,5 & 20,0 & 18,5 \\
\hline Mora s/ companheiro & 68,5 & 80,0 & 81,5 \\
\hline \multicolumn{4}{|l|}{ Trabalho } \\
\hline $\operatorname{Sim}$ & 46,2 & 72,0 & 45,4 \\
\hline Não & 53,8 & 28,0 & 54,6 \\
\hline \multicolumn{4}{|l|}{ Estrato Econômico } \\
\hline Classe A & 0 & 2,0 & 0,8 \\
\hline Classe B & 65,4 & 80,0 & 79,8 \\
\hline Classe C & 34,6 & 18,0 & 19,3 \\
\hline
\end{tabular}


Os resultados apresentados na tabela 2 foi constituído por indivíduos que não dançam, demonstram que, dos 119 praticantes de dança, $36 \%$ frequentam os salões de dança uma vez por semana. Dentre estes, $42 \%$ permanecem por porém pratica outra atividade física sistemática, $50 \%$ se exercitam de duas a três vezes por semana e todos desenvolvem atividades corporais (ginástica, alongamento, caminhada, yoga), num período de uma hora $(56 \%)$.

Tabela 2 - Caracterização da prática da dança e da atividade física. Florianópolis-SC, 2010.

\begin{tabular}{lcc}
\hline \multicolumn{1}{c}{ Variáveis } & $\mathrm{n}$ & $\%$ \\
\hline Grupo da Dança - Frequência & 20 & 16,8 \\
Mensalmente & 43 & 36,1 \\
1 vez por semana & 38 & 31,9 \\
2 a 3x por semana & 18 & 15,1 \\
Mais de 4x por semana & & \\
Tempo nos Salões & 16 & 13,4 \\
Uma hora & 40 & 33,6 \\
Duas horas & 50 & 42,0 \\
Três horas & 9 & 7,6 \\
Mais de quatro horas & & \\
Grupo da Atividade Física - Frequência & 4 & 8,0 \\
1 vez por semana & 25 & 50,0 \\
2 a 3x por semana & 12 & 24,0 \\
4x por semana & 9 & 18,0 \\
Mais de 5x por semana & & 10,0 \\
Duração & 5 & 22,0 \\
30min & 11 & 56,0 \\
30 a 45min & 28 & 12,0 \\
60 min & 6 & \\
Mais de 60 min & & \\
\hline
\end{tabular}

Na tabela 3 observa-se que, de um modo geral, a qualidade de vida pode ser considerada positiva com escores que variam de 70,3 a 58,9 entre seus domínios. O valor superior foi observado no domínio ambiental, e o inferior, no domínio físico. Os participantes que praticam outra atividade que não a dança apresentam melhores resultados no domínio social, com escore médio de 74,3 $\pm 17,9$, e o pior resultado no domínio físico com escore médio de 60,5 $\pm 10,5$. Os subgrupos formados pelos praticantes de dança e os que não realizam atividade física apresentam uma hierarquização semelhante entre os domínios com melhores escores no domínio ambiental $(70,7 \pm 11,2$ e $70,1 \pm 11,3$, respectivamente) e piores escores no domínio físico (57,9 $\pm 9,4$ e $60,7 \pm 11,2$, respectivamente). 
Tabela 3 - Qualidade de vida dos participantes do estudo. Florianópolis-SC, 2010.

\begin{tabular}{lccccccc}
\hline \multirow{2}{*}{ QV } & \multicolumn{2}{c}{ Total } & \multicolumn{2}{c}{ A F } & \multicolumn{2}{c}{ Dança } & \multicolumn{2}{c}{ Não realiza AF } \\
\cline { 2 - 8 } & Escore & Escore & Amp. & Escore & Amp. & Escore & Amp. \\
\hline D. Físico & $58,9 \pm 10,0$ & $60,5 \pm 10,5$ & $39,2-85,7$ & $57,9 \pm 9,4$ & $32,1-78,5$ & $60,7 \pm 11,2$ & $39,2-82,1$ \\
D. Psicológico & $66,2 \pm 9,2$ & $66,2 \pm 9,02$ & $45,8-91,6$ & $66,7 \pm 9,2$ & $41,6-95,8$ & $63,7 \pm 9,1$ & $37,5-83,3$ \\
D. Social & $69,8 \pm 16,2$ & $74,3 \pm 17,9$ & $25,0-100,0$ & $68,06 \pm 15,7$ & $25,0-100,0$ & $69,5 \pm 14,1$ & $41,6-81,3$ \\
D. Ambiental & $70,3 \pm 11,9$ & $69,3 \pm 13,8$ & $35,7-100,0$ & $70,7 \pm 11,2$ & $25,0-100,0$ & $70,1 \pm 11,3$ & $50,0-92,8$ \\
\hline
\end{tabular}

QV: Qualidade de Vida ; D: Domínio; AF: atividade física; Amp: amplitude

De acordo com a tabela 4, a maioria dos participantes teve percepção positiva em relação à finitude. Nomeadamente, o grupo que realiza dança $(80 \%)$ possui uma percepção mais positiva em relação aos demais $(p=0,035)$. O inverso também é verdadeiro, evidenciando que o grupo que apresentou maior ocorrência de percepção negativa foi o composto por sujeitos que não praticam atividade física (39\%). Esta percepção positiva é extensiva aos valores médios calculados em cada um dos fatores (1 a 5), sendo que o fator que se destaca mais positivamente é referente à manutenção da integridade na velhice.

Tabela 4 - Comparação da percepção e domínios da finitude entre os grupos. Florianópolis-SC, 2010.

\begin{tabular}{lcccc}
\hline \multicolumn{1}{c}{ Finitude } & $\begin{array}{c}\text { Não realiza AF } \\
\mathrm{n}=26\end{array}$ & $\begin{array}{c}\text { Atividade Física } \\
\mathrm{n}=50\end{array}$ & $\begin{array}{c}\text { Dança } \\
\mathrm{n}=119\end{array}$ & Valor $\mathrm{p}$ \\
\hline Percepção Positiva $(\%)$ & 61,5 & 64,0 & 79,8 & \\
Percepção Negativa $(\%)$ & 38,5 & 36,0 & 20,2 & \\
Felicidade e velhice $(\bar{X})$ & $2,2 \pm 0,3$ & $2,3 \pm 0,4$ & $2,4 \pm 0,7$ & 0,542 \\
Dependência e velhice $(\bar{X})$ & $2,2 \pm 0,4$ & $2,3 \pm 0,5$ & $2,8 \pm 0,8$ & 0,001 \\
Morte e velbice $(\bar{X})$ & $2,2 \pm 0,8$ & $2,2 \pm 0,7$ & $2,3 \pm 1,2$ & 0,684 \\
Integridade e velhice $(\bar{X})$ & $2,0 \pm 0,7$ & $1,8 \pm 0,6$ & $2,0 \pm 1,2$ & 0,266 \\
\hline
\end{tabular}

AF: Atividade física.

\section{DISCUSSÃO}

Neste estudo, os praticantes de dança e atividade física tiveram a qualidade de vida e a percepção de finitude mais positiva quando comparados com os que não realizam atividade física.

Os resultados evidenciam que a qualidade de vida dos participantes que realizam algum tipo de atividade física é superior quando comparada com a dos não praticantes. Esta percepção de qualidade de vida e saúde ${ }^{21,22}$ se encontra intimamente ligada à prática de atividade física, pois o aumento do nível de atividade exerce impacto positivo na diminuição da morbidade e, eventualmente, da mortalidade da população em geral e na idosa em particular. Quanto mais ativo o idoso, maior sua satisfação com a vida, seu estado de saúde e, consequentemente, melhor sua qualidade de vida. ${ }^{23}$ 
Os benefícios da atividade física no processo de envelhecimento estão relacionados a efeitos psicológicos, tais como melhora do autoconceito, da autoestima, da imagem corporal, diminuição da ansiedade e tensão muscular, minimização da insônia e do consumo de medicamentos, e a socialização. Apresenta também relação significativa entre os níveis de atividade física e o estado de saúde mental, evidenciando menor prevalência de indicadores de depressão e ansiedade. ${ }^{24-26}$ Isso corrobora o presente estudo, que apresentou melhores escores para o domínio psicológico (depressão, ansiedade, entre outros) para os praticantes de atividade física e aqueles que dançam.

Quando se realiza uma comparação entre a percepção dos domínios da qualidade de vida entre os subgrupos, algumas questões emergem. Uma delas se refere a quão bem a amostra se sente em relação à questão ambiental e ao aspecto social, independentemente do subgrupo a que pertence. Por outro lado, os domínios físico e psicológico são, em ordem decrescente, os mais críticos para a amostra. Estes resultados sugerem que, ao refletir sobre as questões referentes à sua qualidade de vida, os indivíduos o fazem considerando não apenas o momento presente de suas vidas, mas em comparação com suas experiências ao longo de seu ciclo vital.

Desta forma, seria compreensível o indivíduo mostrar-se mais satisfeito com o apoio ambiental e de sua rede de relacionamentos, embora consciente da inevitabilidade do declínio físico decorrente do envelhecimento que, por sua vez, se reflete no aspecto psicológico. Mesmo acontecendo um declínio físico, os participantes deste estudo apresentaram maior número de percepção positiva em relação à finitude. Esta percepção positiva é extensiva aos valores médios calculados em cada um dos fatores. Entre os quatro fatores, aquele que congrega uma valoração mais positiva é o referente à manutenção da integridade do sujeito na velhice, o que corresponde a acreditarem que se sentem satisfeitos com suas realizações ao longo da vida, sendo possível, em idade tardia, achar que a vida valeu à pena.
Num estudo objetivando conhecer o significado da velhice e da experiência de envelhecer para os idosos do Estado do Ceará, estes revelaram que a velhice traz muitas perdas, entretanto se sentem realizados com as conquistas pessoais e materiais. ${ }^{27} \mathrm{O}$ estudo ainda revela que a experiência de envelhecer e a velhice, para o grupo, se mostram como acontecimento positivo, comparando-se aos mitos e preconceitos oriundos do meio urbano. Em estudo com o intuito de conhecer a opinião de idosos do Distrito Federal a respeito do envelhecimento, utilizando o mesmo instrumento, os idosos concordam com esses resultados, ${ }^{28}$ sendo encontrada a mesma semelhança. ${ }^{29} \mathrm{Na}$ Região Centro Oeste do Brasil, as principais preocupações dos idosos são mostrar que não perderam sua identidade por ficarem idosos, e muitas vezes não se sentem envelhecidos, mesmo com a idade cronológica avançada. ${ }^{30}$

A possibilidade de se tornarem dependentes na velhice é o fator que mais preocupa os participantes, sendo maior entre os que dançam - o que tem fundamento, pois são indivíduos ativos e se preocupam com o fato de deixarem de fazer certas atividades, até mesmo a dança. Estes resultados são congruentes com os obtidos na percepção da qualidade de vida, pois os domínios físicos e psicológicos foram os que apresentaram menores escores. Considerando-se a média de idade da amostra, é natural que alguns já apresentem sinais que prenunciam perda em decorrência do processo de envelhecimento.

Embora exista uma tendência a perspectivas positivas em relação à velhice pessoal, e que o ser humano acredita que esta fase propicia sentimentos de integridade, sendo possível ser feliz nesse período, eles também creem que a velhice pode trazer dependência, morte e solidão. $\mathrm{E}$ ainda, alguns manifestam que é melhor morrer cedo do que sentir a angústia e a solidão da velhice. ${ }^{31}$ Em estudo utilizando o mesmo instrumento, os idosos declararam possuir medo da debilidade física. ${ }^{28}$ Esses resultados correspondem a alguns estudos, ${ }^{30}$ nos quais se 
evidenciou que ter saúde é essencial porque os permite manter autonomia sobre suas vidas, mas evitam falar sobre a finitude do ser humano. As situações relacionadas a perdas, especialmente aquelas que remetem à finitude, estão fortemente presentes na vida de idosos. ${ }^{32}$

Assim como o nascer, a morte faz parte do processo de vida do ser humano. Algo extremamente natural do ponto de vista biológico, entretanto, o ser humano caracterizase também pelos aspectos simbólicos, ou seja, pelo significado ou pelos valores que ele imprime aos fatos. O sentido da morte, por isso, varia necessariamente no decorrer da história e entre as diferentes culturas humanas. ${ }^{33}$

Em cada sociedade existe um significado atribuído à morte. Inicialmente, esse significado é externo ao indivíduo, pertencendo à cultura. À medida que esse significado é internalizado, transforma-se num instrumento subjetivo da relação do indivíduo consigo mesmo. E igualmente, o significado externo adquire um sentido pessoal para o indivíduo. Através da atividade e das relações sociais estabelecidas com o meio, o indivíduo internaliza esses conteúdos e significados a partir de sua própria experiência e história de apropriações (ou seja, sua subjetividade). Dessa maneira, o conteúdo que tinha um significado externo passa por uma mediação psíquica e adquire um sentido pessoal, singular, único para cada pessoa. ${ }^{33}$

Uma visão mais positiva do envelhecimento pode auxiliar os indivíduos a alcançarem e manterem níveis mais altos de funcionamento na medida em que envelhecem. ${ }^{34}$ Os mais velhos, que têm uma autopercepção positiva sobre o envelhecimento, parecem viver mais que aqueles com visão mais negativa, ou seja, uma atitude positiva em relação à velhice e ao desejo de uma vida longeva contribui para que se viva mais e melhor. ${ }^{35}$

Para alguns estudiosos, a finitude deve ser amplamente discutida, pois a consciência crítica e abordagem do tema proporcionam ao idoso um processo de envelhecimento de forma tranquila com uma maior compreensão e aceitação do envelhecimento. ${ }^{7,8}$

Para desenvolver este estudo, foram encontradas algumas limitações, tais como não ter a possibilidade de constituir uma amostra com o mesmo número de não praticantes de atividade física e também de praticantes de outras modalidades de atividade física além da dança. Nos centros de convivência onde os dados foram obtidos, a maioria frequentava os salões de dança de salão ou não se encontrava no momento das coletas, ou ainda se recusou a participar do estudo.

Da mesma forma, a amostra apresentou muitas mulheres, não sendo possível trabalhar os dados por sexo para comparação dos integrantes. $\mathrm{Na}$ literatura, vemos que existe diferença entre os sexos nas mais diversas categorias, e seria interessante demonstrar se existe essa diferença na percepção de finitude e em como a atividade física influencia esta variável.

\section{CONCLUSÃO}

Os resultados obtidos neste estudo sugerem uma qualidade de vida positiva, sobretudo nos domínios psicológico e ambiental. Entre os subgrupos, o domínio social destaca-se positivamente entre os praticantes de atividade física e os domínios psicológico e ambiental, entre os praticantes de dança nos salões. A percepção do envelhecimento também se mostra positiva entre os integrantes da amostra.

De acordo com os resultados, quem realiza atividade física tende a aceitar e lidar mais facilmente com as perdas e a proximidade da morte. Curiosamente, o grupo dos que não praticam atividade física encara mais facilmente este processo, quando comparado com os que praticam dança, principalmente quanto à possibilidade de associação entre dependência e velhice. 


\section{REFERÊNCIAS}

1. Ibge. Instituto Brasileiro de Geografia e Estatística. [Acesso em: 20 de outubro de 2009]. Disponível em $<$ http:// www. ibge.gov.br>

2. Wong LR, Carvalho JA. O rápido processo de envelhecimento populacional do Brasil: sérios desafios para as políticas públicas. Rev Bras Estudos de População, 2006, 23(1).

3. Kaufmann IG. Perdas, perdas, perdas... Consequências do envelhecimento! Relato de um processo psicoterapêutico com início em atendimento em grupo. III Congresso IberoAmericano de Psicogerontologia 2009.

4. Lopes R. Os velhos ainda precisam ser "Indignos"? In: Corte B, Mercadante EF, Arcuri IG, organizador. Complex(idade). Velhice Envelhecimento. Ed Vetor, SP; 2005.

5. Schramm FR. Morte e finitude em nossa sociedade: implicações no ensino dos cuidados paliativos. Rev Bras Cancerol, 2002, 48(1), 17-20.

6. Rego S, Palácios M. A finitude humana e a saúde pública. Cad Saúde Púb, 2006, 22(8), 755-1760.

7. Frumi C, Celich KLS. O olhar do idoso frente ao envelhecimento e à morte. Rev Bras Ciên Envelhec Hum 2006, 3, 92-100.

8. Zinn GR, Gutierres BA. O processo de envelhecimento e sua relação com a morte: percepção do idoso hospitalizado em unidade de cuidados semi-intensivos. Est Interdisc envelhecimento. 2008, 13(1), 79-93.

9. Moraes $\mathrm{H}$, et al. $\mathrm{O}$ exercício físico no tratamento da depressão em idosos: revisão sistemática. Rev Psiqu Rio Grande do Sul, 2007, 29(1), 70-9.

10. Rocha, MD. Dança de Salão: instrumento para a qualidade de vida. Mov Percepção, 2007, 7(10).11. Silva MC. Senhorita hoje, senhora amanhã: análise do envelhecimento e da dança como exercício físico. São Paulo: $5^{a}$ mostra acadêmica UNIMEP, 2007.

11. Keyani P, et al. Dance along: supporting positive social exchange and exercise for the elderly through dance. Portland:Presented in Conference on Human Factors in Computing Systems 2005 [s.n.], 1541-44.

12. Gobbo DE, Carvalho D. A dança de salão como qualidade de vida para a terceira idade. Rev Elet de Educ Fís. 2005

13. Siqueira MD. Sociabilidade e Envelhecimento feminino nos Bailes de Dança de Salão em Fortaleza. Universidade Federal do Ceará, 2009.
14. Oliveira RG, Tolocka RE. Inclusão social e pessoas que participam de bailes em uma instituição de longa permanência para idosos. Rev Educ Fís UEM. 2009, 20(1), 85-96.

15. Loparic, Z. A ética da finitude. Belém: Editora universitária; 1994.

16. Bernieri J, Hirdes A. O preparo dos acadêmicos de enfermagem brasileiros para vivenciarem o processo morte-morrer. Texto \& Contexto Enferm, 2007, 16(01), 89-96

17. Associação Brasileira de Empresas de Pesquisa - ABEP. Critério de Classificação Econômica Brasil. 2008. [Acesso em 02 de novembro de 2008]. Disponível em <http://www.abep.org>.

18. Fleck MPA, et al. Aplicação da versão em português do instrumento Whoqol abreviado. Rev Saúde Púb 2000, 34(2), 178-183.

19. NERI, A. L. O inventário Sheppard para medida de atitudes em relação à velhice e sua adaptação para o português. Est Psicol 1986, 3 (1 e 2), 23-42.

20. Mota J, et al. Atividade física e qualidade de vida associada à saúde em idosos participantes e não participantes em programas regulares de atividade física. Rev Bras Educ Fís e Esp. 2006, 20(3), 219-225.

21. Silva RS, et al. Atividade física e qualidade de vida. Ciên Saúde Col, 2010,15(1), 115-120.

22. Pereira RJ, et al. Contribuição dos domínios físico, social, psicológico e ambiental para a qualidade de vida global de idosos. Rev Psiquiatria. 2006, 28(1), 27-38.

23. Matsudo SM, Matsudo VKR, Barros Neto TL. Efeitos benéficos da atividade física na aptidão física e saúde mental durante o processo de envelhecimento. Rev Bras Ativ Fís e Saúde. Londrina, 2000, 5(2), 60-76.

24. Stella F, et al. Depressão no Idoso: Diagnóstico, Tratamento e Benefícios da Atividade Física. III Congresso Internacional de Educação Física e Motricidade Humana. 2003 abr/mai.

25. Alves RV, et al. Aptidão física relacionada à saúde de idosos: influência da hidroginástica. Rev Bras Med Esp 2004, 10(1).

26. Freitas MC, Queiroz TA, Sousa JAV. O significado da velhice e da experiência de envelhecer para os idosos. Rev Esc Enfermagem 2010, 44(2), 407-12.

27. Zanon CBFM. A educação e a intergeracionalidade na perspectiva da inserção social do idoso: desafios e possibilidades. [Dissertação]. Brasília: Universidade Católica de Brasília; 2006. 
28. Resende MC, Neri AL. Ajustamento psicológico e perspectiva de velhice pessoal em adultos com deficiência física. Psicol estud, 2009 14(4),767-76.

29. Silva MG, Boemer MR. The experience of aging: a phenomenological perspective. Rev Lat Am. Enfermagem, 2009 17(3), 380-86.

30. Resende MC. Ajuste Psicológico, perspectiva do envelhecimento pessoal e satisfação e a vida em adultos e idosos com deficiência física. [tese]. Campinas Faculdade de Educação da UNICAMP, 2006.
31. Fortes-Burgos ACG, Neri AL, Cupertino APFB. Eventos de vida estressantes entre idosos brasileiros residentes na comunidade. Est Psicol. 2009, 14(1), 69-75.

32. Combinato DS, Queiroz MS. Morte: uma visão psicossocial. Estud. Psicol. (Natal) [online]. 2006, vol.11, n.2, pp. 209-16.

33. Seeman TE. Health promoting effects of friends and family on health outcomes in older adults. Am J Health Prom, 2000, 14, 362-70.

34. Levy BR, et al. Longevity Increased by Positive Self-Perceptions of Aging. J Person Soc Psych 2002, 83(2), 261-270. 